\title{
Freshness-driven Adaptive Caching for Dynamic Content
}

\author{
Wen-Syan Li Oliver Po Wang-Pin Hsiung $\quad$ K. Selçuk Candan Divyakant Agrawal \\ C\&C Research Laboratories - Silicon Valley, NEC USA, Inc. \\ 10080 North Wolfe Road, Suite SW3-350, Cupertino, California 95014, USA \\ Email:\{wen,oliver,whsiung,candan,agrawal\}@ccrl.sj.nec.com
}

\begin{abstract}
With the wide availability of content delivery networks, many e-commerce Web applications utilize edge cache servers to cache and deliver dynamic contents at locations much closer to users, avoiding network latency. By caching a large number of dynamic content pages in the edge cache servers, response time can be reduced, benefiting from higher cache hit rates. However, this is achieved at the expense of higher invalidation cost. On the other hand, a higher invalidation cost leads to a longer invalidation cycle (time to perform the invalidation check on the pages in caches) at the expense of freshness of cached dynamic content. In this paper, we propose a freshness-driven adaptive dynamic content caching technique, which monitors response time and invalidation cycle length and $d y$ namically adjusts caching policies. We have implemented the proposed technique within NEC's CachePortal Web acceleration solution. The experimental results show that the proposed technique consistently maintains the best content freshness to users. The experimental results also show that even a Web site with dynamic content caching enabled can further benefit from deployment of our solution with improvement of its content freshness up to 10 times especially during heavy traffic.
\end{abstract}

Keywords: dynamic content, Web acceleration, freshness, response time

\section{Introduction}

Response time and content freshness are essential to ecommerce Web sites. Slow response times and down times can be devastating for e-commerce sites as reported in a study by Zona Research[1] on the relationship between Web page download time and user abandonment rate. The study shows that $30 \%$ of users will leave a Web site (i.e. abandonment rate) if the download time is around 7 seconds and the abandonment rate can reach $70 \%$ as download times exceed 12 seconds. This study clearly establishes the importance of fast response times to an e-commerce Web site to retain its customers.

For many e-commerce applications, Web pages are created dynamically based on the current state of a business, such as product prices and inventory, stored in database systems. This characteristic requires e-commerce Web sites to deploy and integrate Web servers, application servers, and database systems at the backend. A basic system architecture of database-driven Web sites consists of the following components: (1) a Web server (WS) which receives user requests and delivers the dynamically generated Web pages; (2) an application server (AS) that incorporates all the necessary rules and business logic to interpret the data and information stored in the database. AS receives user requests for HTML pages and depending upon the nature of a request may need to access the DBMS to generate the dynamic components of the HTML page; and (3) a database management system (DBMS) to store, maintain, and retrieve all the necessary data and information to model a business.

When the Web server receives a request for dynamic content, it forwards the request to the application server along with its request parameters (typically included in the URL string). The Web server communicates with the application server using URL strings and cookie information, which is used for customization, and the application server communicates with the database using queries. When the application server receives such a request from the Web server, it processes it and it may access the underlying databases to extract the relevant information needed to dynamically generate the requested page.

To improve the response time, one option is to deploy network-wide caches so that a large fraction of requests can be served remotely rather than all of them being served from the origin Web site. Applying caching solutions for Web applications and content distribution has received a lot of attention in the Web and database communities[2, 3, 4, 5, 
$6,7,8,9,10]$. These provide various solutions to accelerate content delivery as well as techniques to assure the freshness of the cached pages. Note that since Web content is delivered through the Internet, the content freshness can only be assured rather than being guaranteed.

For the Web sites that are dynamic content caching enabled, by caching more dynamic contents in edge cache servers, response time can be improved through the benefit of higher cache hit rates and low network latency. However, in a database-driven Web site, databases must be monitored so that the cached pages which are impacted by database content changes can be invalidated or refreshed in a timely manner. As a result, caching a large number of Web pages at edge cache servers to yield fast response time are achieved at the expense of longer invalidation cycles. Thus, it requires more computational resources and time to complete invalidation checking. On the other hand, when an invalidation cycle is long, the freshness of cached pages can not be maintained at a desirable level.

In this paper, we focus on the issue of how to maintain the best content freshness that can be assured for a given set of user request rate and database update rate. We propose an adaptive dynamic content caching technique that monitors response time and invalidation cycle as a feedback for dynamic adjustment of caching policy. By considering the trade-off of invalidation cycle and response time, the proposed technique maintains the best content freshness to the users.

The rest of this paper is organized as follows. In Section 2, we give an overview of our proposed solution. In Section 3 we describe the dynamic content invalidation schemes used in NEC's CachePortal. In Section 4, we describe dependency between user request response time and invalidation cycles. In Section 5 we propose a caching policy for balancing response time and invalidation cycles for maintaining freshness. We also present experimental results to demonstrate the effectiveness of our proposed technique for ensuring content freshness. Section 6 summarizes related work and section 7 concludes the paper.

\section{Problem Statement and Solution Overview}

In this section, we present the problem formulation and give an overview of our proposed solution. We start with the description of an e-commerce Web site system architecture that is dynamic content caching enabled.

Figure 1 shows the architecture and data flows of a database-driven e-commerce Web site where NEC's CachePortal technology[2, 3, 10] is deployed. The architecture is very similar to that of typical e-commerce web sites, except for the two CachePortal components, Sniffer and Invalidator. The CachePortal technology enables dynamic content caching by utilizing Sniffer to derive

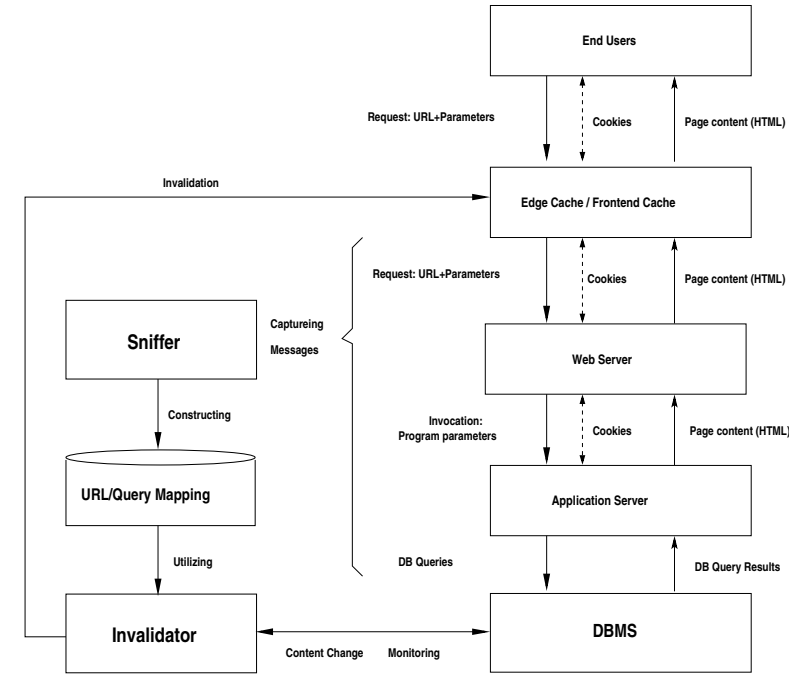

Figure 1. A Database-Driven E-Commerce Site with Dynamic Content Caching Enabled

URL/DB Query Mapping, the relationships between cached pages and the database contents that are used to generate these pages. The Invalidator is responsible for monitoring database changes by scanning database update log. The database changes are monitored periodically by the invalidator and then it performs invalidation checking process to identify the cached pages which are impacted by the database changes during the current period.

In this configuration, user requests are directed to the edge cache that is close to the requesting users. If there is a cache hit, the requested content will be delivered to the users from the cache. Otherwise requests are forwarded to the origin Web server. Note that in our implementation, edge caches behave as both reverse proxies (if there is a hit) and proxies (if there is a miss so that the requests are forwarded). We assume that the proxy servers are close to the users so that the network latency between the cache servers and the users can be disregarded compared with the network latency between the cache servers and the origin Web sites.

Let us define the main three terms that have impact on the freshness of delivered contents as follows:

Response time at the edge cache servers: This is the round trip time for requests that are served at the edge cache servers (as a result of a cache hit). The response time from edge caches is expected to be extremely fast.

Response time at the origin Web sites: This is the round trip time for requests that are served at the origin Web servers (as a result of a cache miss).

Invalidation cycle: This is the time required to process invalidation checks for all the pages in the cache servers. 


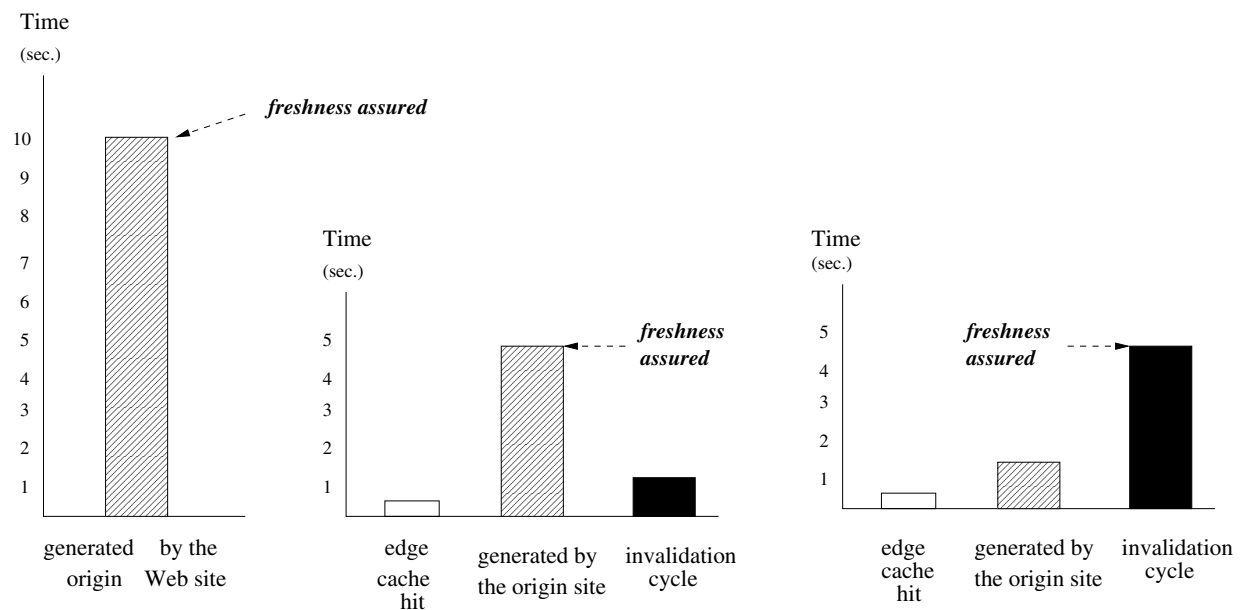

(a) w/o dynamic content caching (b) with dynamic content caching enabled (c) with dynamic content caching enabled

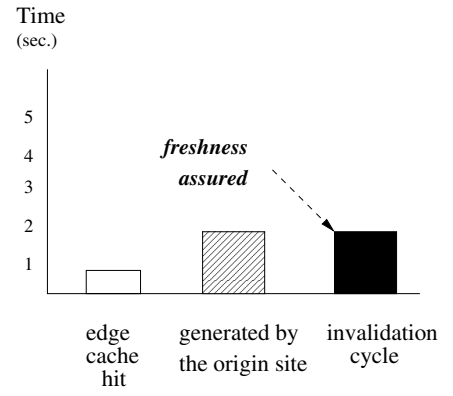

(d) deploying freshness-driven adaptive dynamic content caching

Figure 2. Response Time and Freshness of Delivered Content by Various System Architectures

The invalidation cycle is impacted by the following factors: (1) the update rates in database systems; (2) the number of pages in the cache servers; and (3) the correlation between the pages in the cache servers (in term of the query statements used to generate these pages).

For Web sites that do not deploy any dynamic content caching solution, all pages need to be dynamically generated at the origin Web site. The content freshness such system can assure is the response time at the Web sites. For example, if the response time for a request at a Web site is 10 seconds on the average the Web page can be fresh, but since the database content can change after the Web page is generated, the assured freshness of the page (i.e. age) is 10 seconds!

For Web sites that deploy dynamic content caching solutions, some pages are dynamically generated at the origin Web site but majority of the pages are delivered from the cache server. Since the database content changes need to be reflected to the pages in the cache server, invalidation check needs to be performed periodically or on demand to ensure content freshness. In these Web sites, the content freshness that can be assured is the maximum of (1) the response time from origin Web sites, (2) the response time from edge caches, and (3) the length of the invalidation cycle. Note that since the response time from edge caches is much faster than the response time from either the Web sites or the length of invalidation cycles, the content freshness that can be assured is the larger of the response time from origin Web sites and the length of the invalidation cycle.

Depending on the caching policy, traffic patterns, cache hit rates, server load, and database update rates, sometimes the response time at origin Web sites can be much longer than the invalidation cycle time. In Figure 2(b), we show a system configuration that caches few pages. As a consequence, it is expected to have a short invalidation cycle; at the expense of slow response time when there is a cache miss since the Web site is carrying a heavy load. On the other hand, the system configuration in Figure 2(c) has a different caching policy and caches a large number of Web pages at its edge caches. This configuration provides fast response time for all requests. When there is a cache hit, the response time is naturally very fast. When there is a cache miss, the response is still fast as the Web site has a lighter work load since most of the requests are served directly from the edge cache servers. However, this configuration and its caching policy has potentially low content freshness since it will take much longer time to complete the necessary invalidation check for all the pages in the cache servers.

The configuration in Figure 2(d), on the other hand, has a caching policy tuned so that the response time at the origin Web site is very close to the length of the invalidation cycle. This configuration has several advantages. First, it allows the best content freshness among these system architectures; with or without dynamic content caching solutions. Second, it also yields fast response times. Note that the configuration in Figure 2(d) does not choose to provide lower response times, by caching additional pages to increase the cache hit rate and consequently improve the response time. However caching additional pages would lead to longer invalidation cycles and consequently reduce the level of content freshness.

From this above example, we can see that the freshness of requested pages can be ensured at the highest level when the hit rate is tuned to a point where the response time from the origin site and invalidation cycle for the cached pages 
are the same. In this paper, we propose a freshness-driven adaptive dynamic content caching technique. The proposed technique aims at maintaining the best content freshness that a system configuration can support and assure. Our technique does not attempt to maintain the lowest average response time nor average content freshness. Instead it assures good content freshness: the delivered content is either fresh or not older than a threshold (i.e. the assured content freshness) given by the system .

\section{Invalidation Checking Process}

In this section, we describe the invalidation scheme used in the proposed system architecture. Assume that the database has the following two tables: Car (maker, model, price) and Mileage (model, EPA). Say that the following query Query 1 has been issued to produce a Web page, $U R L 1$ :

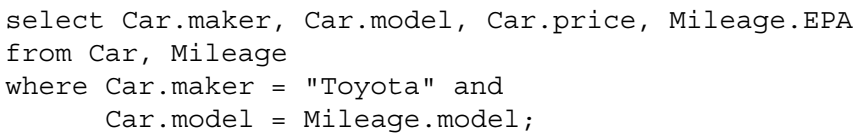

Let a new tuple (Toyota, Avalon, $\$ 25,000$ ) be inserted into the table Car. Since Query 1 accesses two tables, we first check if the new tuple value can satisfy the condition associated with only the table Car stated in Query 1 . If it does not satisfy, we do not need to test the other condition and we know the new insert operation does not impact the query result of Query 1 and consequently $U R L 1$ does not need to be invalidated or refreshed.

If the newly inserted tuple does satisfy the condition associated with the table $C a r$, we cannot determine whether or not the query result of Query 1 has been impacted unless we check the rest of the condition associated with the table Mileage. To check whether or not the condition Car.model $=$ Mileage.model can be satisfied, we need to access the table Mileage. To check this condition, we need to issue the following query, Query 2 , to the database:

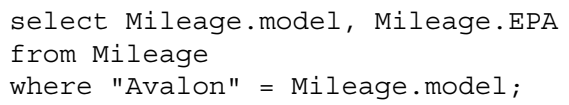

If the result of Query 2 is non-empty, the query result for Query 2 needs to be invalidated. The queries, such as Query 3 , that are issued to determine if certain query results need to be invalidated are referred as polling queries.

Next, assume that we observe the following queries, Query3 and Query4, in the URL/database query map to generate user requested pages:

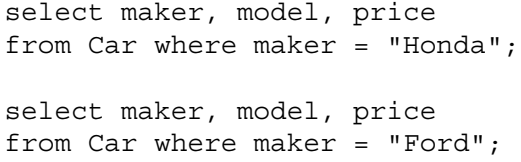

we can derive a query type, Query_Type 1 as:

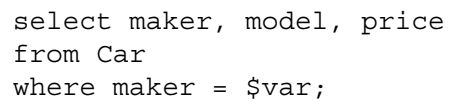

Therefore, multiple query instances can have the same bound query type; and, multiple bound query types may have the same query type. We can create a temporary table Query_Type1_Inst to represent the above two query instances as follows:

$\begin{array}{cc}\text { QUERY_ID } & \text { QUERY_INSTANCE } \\ \text { Query3 } & \text { Honda } \\ \text { Query4 } & \text { Ford } \\ \end{array}$

Let us also assume that the following four tuples are inserted to the database (refer to this table as Delta):

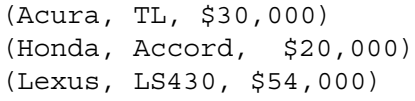

We can consolidate a number of invalidation checks into a more "compact" form through a transformation. For example, a single polling query, Query 5 , can be issued as follows:

select Query_Type.QUERY_ID

from Car, Query Typel Inst, Delta

where Delta.Maker = Query_Type1_Inst.QUERY_INSTANCE;

Query_Type1_Inst.QUERY_ID is a list of query results that need to be invalidated. In this example, Query 3 will be invalidated. We summarize important characteristics of the consolidated invalidation checking scheme as follows: (1) the invalidation cycle is mainly impacted by the number of cached query types since that it determines the number of polling queries executed for invalidation checking; and (2) database update rates and the number of query instance per cached query type would have relatively lower impact on the invalidation cycle since they only increase query processing cost.

\section{Dependency between Response Time and In- validation Cycle}

In this section, we examine the dependency between the response time and the invalidation cycle length. We have conducted experiments to verify this dependency. We first describe the general experiment setup that consists of databases, application servers, and network infrastructure that are used in the experiments.

\subsection{General Experimental Setting}

The experimental setting of our evaluations is as follows: 
- We used two heterogeneous networks that are available in the NEC's facility in Cupertino, California: one is used by the C\&C Research Laboratories (referred to as CCRL) and the other one is used by cacheportal.com (referred to as CP). Users and cache servers (edge caches in this setting) are located in CCRL while the Web server, application server, and DBMS are located in CP. The average number of hubs between CCRL and $\mathrm{CP}$ is 15 . The average throughput measured for CCRL-CP, CP-CP, and CCRL-CCRL connections are 84.7, 682.4 Kilobytes/second, and 482.4 Kilobytes/second respectively. The average round trip time on CCRL-CP, CP-CP, and CCRL-CCRL connections are $321 \mathrm{~ms}, 1 \mathrm{~ms}$, and $2 \mathrm{~ms}$ respectively. To summarize, connectivity within the same network is substantially better than that across the Internet and there is network latency between two networks.

- Oracle 9i is used as the DBMS and is on a dedicated machine. The database contains 7 tables with $1,000,000$ rows each. The database update rate can be set as 5,000, 10,000, and 15,000 rows per table per minutes.

- The maximum number of pages that can be cached is $2,000,000$ pages. These pages are generated by queries that can be categorized into 200 query types. Assuming uniform distribution, there are 10,000 pages (query instances) per query type.

- BEA WebLogic 6.1 is used for the WAS, and Apache is used as the edge cache server and they are located on dedicated machines. Each machine is a Pentium III 700Mhz single CPU PC running Redhat Linux 7.2 with $1 \mathrm{~GB}$ of memory.

- The invalidation mode is set as "continuous". In the continuous invalidation mode, the invalidator will fetch a new block of database update log to start processing immediately after it completes an invalidation check cycle.

\subsection{Correlation between Number of Query Types and Cache Hit Rates}

The problem of cache replacement has been extensively studied. Many algorithms have been proposed for general purpose caching, such as LRU and LFU. Some variations of these are designed specifically for cache replacement of Web pages. However, in the scope of dynamic caching for a Web site, cache invalidation rate is an important factor since a high invalidation rate will lead to a potentially high cache miss rate in the future. As a result of the high miss rate, the WAS and DBMS have to handle a higher load to generate Web pages. We have developed a dynamic content cache

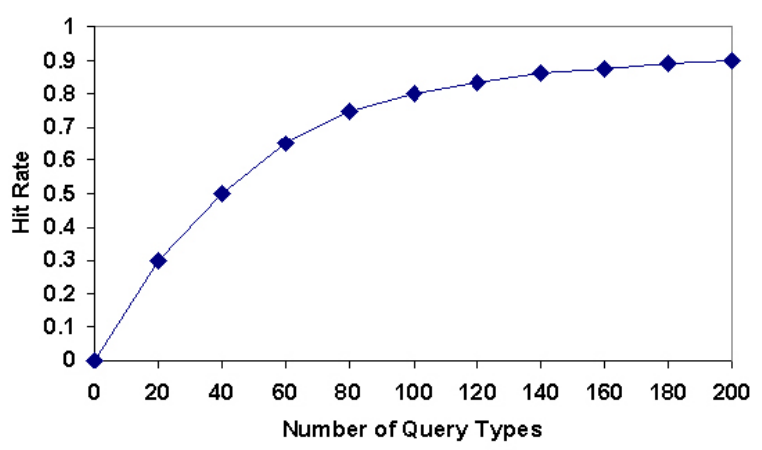

\section{Figure 3. Correlation between Number of Query Types and Cache Hit Rates}

replacement algorithm [11] that takes into consideration (1) user access patterns, (2) page invalidation pattern, and (3) temporal locality of the requests. The caching priority of each page is re-calculated periodically. The caching priority of a page during a time period $t$, Caching priority $(t)$, is calculated as

$(1-\alpha) \times \frac{\text { access_rate }}{\text { invalidation_rate }}+\alpha \times$ Caching_priority $(t-1)$

where $\alpha$ is the temporal decay factor whose value is between 0 and 1 . The intuition behind this formula is that it estimates the average number of accesses for a page between any two successive invalidations. The higher this number the larger the benefit to keep this page in the cache. After we calculate the caching priority for each page, we then calculate the caching priority of each query type in a similar way: we calculate their caching priority by aggregating the access rate and invalidation rate for all pages generated by the same query type.

Consequently, we are able to select and cache only a small number of query types and pages generated by these query types, but maintain a high hit rate at the caches. Figure 3 shows the correlation between the number of cached query types and the cache hit rates. As we can see in the figure, when we select and cache 20 query types (10\% of all query types), the cache hit rate is close to $30 \%$. And, when we select and cache 100 query types (50\% of all query types), the cache hit rate is close to $80 \%$.

\subsection{Effects of Request Rates and Numbers of Query Types on Invalidation Cycles}

As the summary in Section 3 states, the number of cached query types has the major impact to the invalidation cycle since it directly determines the number of polling queries that must be executed. In the experiments, we vary the number of cached query types and measure the invalidation cycle time when the number of cached query types is $20,60,100,140$, and 180 respectively. Figure 4(a) 


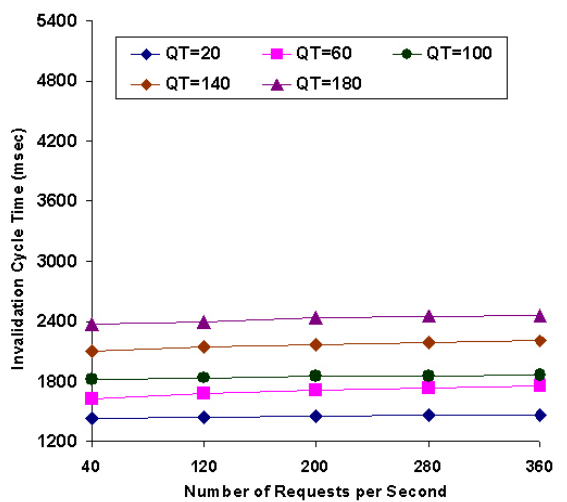

(a)

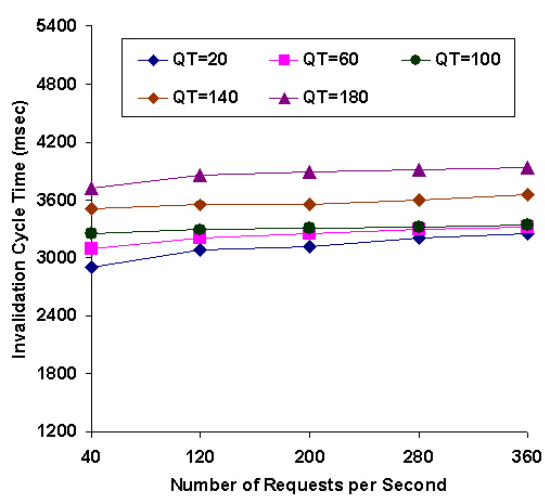

(b)

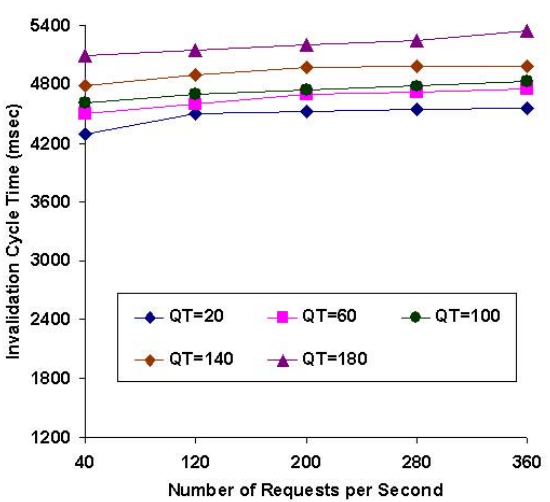

(c)

Figure 4. Effects of Request Rates and Numbers of Query Types on Invalidation Cycles (Database Update Rate $=$ (a) 5,000; (b) 10,000; and (c) 15,000 Tuples per Table per Minute)

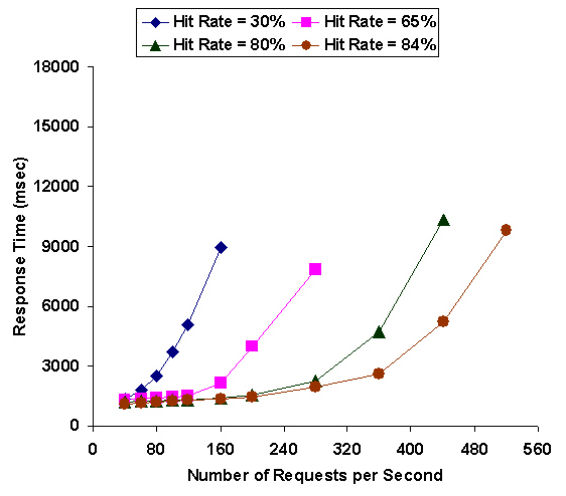

(a)

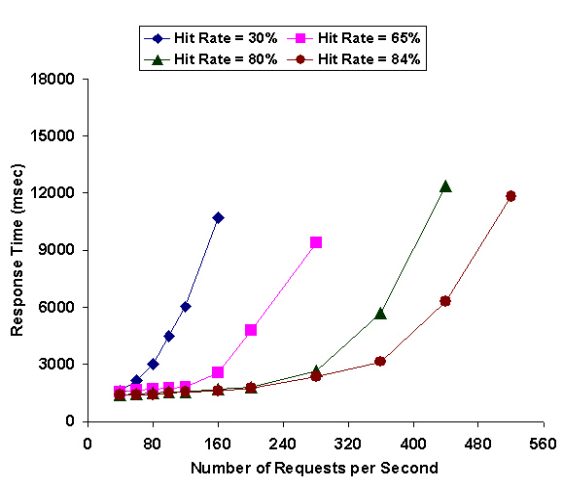

(b)

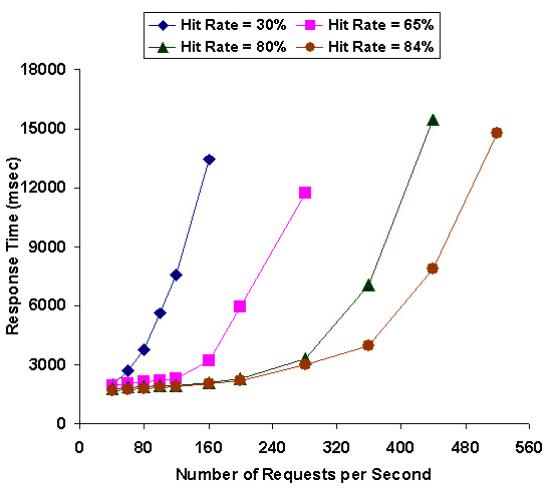

(c)

\section{Figure 5. Effects of Request Rates and Cache Hit Rates on Request Response Time (Database Update Rate $=$ (a) 5,000; (b) 10,000; and (c) 15,000 Tuples per Table per Minute)}

shows the experimental results that illustrate the effects of the number of cached query types on the invalidation cycle. As the number of cached query types increases, so does the length of the invalidation cycle. Note that the polling query for each cached query type takes two temporary tables to join: the query instance table and the database change table. When the invalidation cycle is shorter, the database change table is smaller accordingly when query instance table remains the same. As a result, the polling queries for the shorter invalidation cycle have lower query processing cost. Figure 4(a) also shows that the request rates have limited impact on the invalidation cycle although a higher request rate does put a heavier load on the DBMS where the invalidation checks are performed.

\subsection{Effects of Request Rates and Cache Hit Rates on Request Response Time}

The next experiment we conducted is to measure the effects of cache hit rates and request rates on the response time. In this figure, we plot the response time with respect to a set of different request rates (i.e. from 40 requests per second to 680 requests per second) and different cache hit rates (i.e. $30 \%, 65 \%, 80 \%, 84 \%$, and $88 \%$ ). Note that since the cache hit rates are directly impacted by the numbers of cached query types, the cache hit rates of 30\%, 65\%, $80 \%$, $84 \%$, and $88 \%$ correspond to the setup where the numbers of cached query types are $20,60,100,140$, and 180 respectively. The setup for the experiments in Figures 5(a) and 4(a) are the same, but we measure invalidation cycles in Figure 4(a) and measure request response time in Figure $5(\mathrm{a})$. 
The experimental results in Figure 5(a) indicate that request response time is more sensitive to the request rate than the invalidation cycle length. When the request rate reaches a certain threshold, the response time increases sharply.

\subsection{Effects of Database Update Rates on Invalida- tion Cycles and Request Response Time}

The next experiment we conducted is to measure the effects of database update rates on cache invalidation cycles and request response time. We repeated the experiments in Sections 4.3 and 4.4 by varying the database update rate from 5,000 tuples per table per minute to 10,000 and 15,000 tuples per table per minute. Our observations based on the experimental results are as follows:

- The increase in the invalidation cycle length is directly proportional to the increase in the database update rate. This is expected as the polling query processing costs are proportional to the database update rate, when the number of query instances is fixed. This is illustrated in Figures 4(a), 4(b), and 4(c).

- The database update rates have limited impact on the request response time. For example, when the database update rate increases from 5,000 tuples per table per minute to 10,000 tuples per table per minute, the request response time increases by about $10 \%$. Although a higher database update rate puts a heavier load on the DBMS, the major portion of request response time is network latency rather than the server latency. As a result, the effects of database update rates to the request response time is very limited. This is illustrated in Figures 5(a), 5(b), and 5(c).

\section{Freshness-driven Adaptive Dynamic Con- tent Caching}

In this section, we describe the proposed freshnessdriven adaptive dynamic content caching followed by the discussion and analysis of the experimental results.

\subsection{Adaptive Caching Policy}

To achieve the best assured dynamic content freshness, we need to tune the caching policy so that the response time is close to the invalidation cycle in an equilibrium point. However, the response time and invalidation cycle can only be improved at the expense of each other. In Section 4, we found that response time can be impacted by (1) network latency, (2) request rates, (3) database update rates, (4) invalidation cycle (frequency), and (5) cache hit rates. Network latency, request rates, and database update rates depend on the network infrastructure and application characteristics and hence can not be controlled. However, we can "control" (i.e. tune) the frequency of invalidation cycles. Furthermore, the cache hit rate can be controlled by the number of cached query types. We can derive the following adaptive caching policy for maintaining request response time and invalidation cycle close to an equilibrium point:

- If the response time is larger than the length of the invalidation cycle, we can lower the request response time by increasing the number of cached query types until the request response time and invalidation cycle reach an equilibrium point.

- If the invalidation cycle is longer than the request response time, we can lower the invalidation cycle by decreasing the number of cached query types until the request response time and invalidation cycle reach an equilibrium point.

Note that when the invalidation cycle is longer than the response time and we shorten the length of the invalidation cycle by decreasing the number of cached query types, the increase of request response time and the decrease of invalidation cycle will occur at the same time. As a result, an equilibrium point can be achieved fast. Similarly, when we increase the number of cached query types, the decrease of request response time and the increase of invalidation cycle will occur at the same time.

In the current implementation, the adaptive caching policy is deployed at the edge server. The response time is measured at the cache server assuming that the round trip between users and the cache server (i.e. also functioning as a user side proxy) is negligible.

\subsection{Experiments}

We conducted a series of experiments to evaluate the proposed freshness-driven adaptive dynamic content caching technique. In these experiments, we created setups by varying request rates and database update rates (every $10 \mathrm{~min}$ utes). We then observed the effects of our freshness-driven adaptive caching technique on maintaining the best freshness that can be assured for a given system configuration and setup. In Figure 6, we plot the response time as a dotted line and invalidation cycle as a solid line. The numbers next to the dotted line are the number of cached query types being cached.

We observe that the sudden changes of the request rate will cause temporary imbalance of response time and invalidation cycle. Especially the response time is very sensitive to the changes of request rates. As time moves on, the freshness-driven adaptive caching technique makes necessary adjustments to the number of cached query types; and 
Freshness-driven Adaptive Dynamic Content Caching

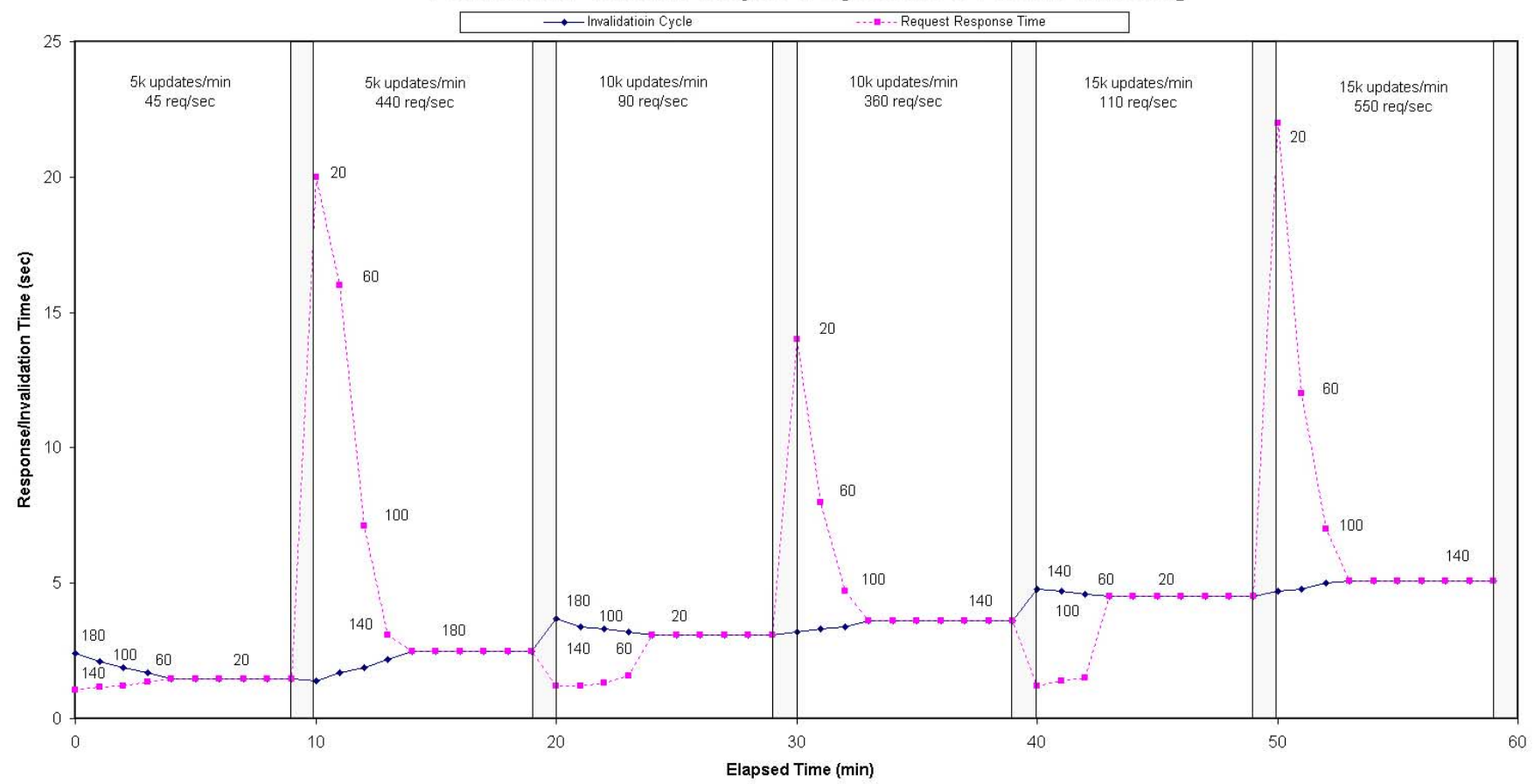

Figure 6. Impact of Adaptive Caching on Content Freshness

this impacts the cache hit rate and the response time. As time elapses, the response time and invalidation cycle are shifted back to a new equilibrium point, which supports the best content freshness that can be assured.

From Figure 6, we can see that the request response time can be adjusted quickly since it is very sensitive to the cache hit rate. We also observe that the invalidation cycle can not be adjusted as quickly. These observations are consistent with our experimental results in Section 4.

Next we compare the content freshness that can be assured by four different system configurations as follows:

1. a system configuration that does not deploy any dynamic content caching solution;

2. a system configuration that is dynamic content caching enabled but that does not employ the proposed freshness-driven adaptive caching technique. 60 query types are cached;

3. a system configuration that is dynamic content caching enabled but that does not employ the proposed freshness-driven adaptive caching technique. 140 query types are cached;

4. a system configuration that is dynamic content caching enabled and that employs the proposed freshnessdriven adaptive caching technique.

In Figure 7 we plot the larger of the response time and the invalidation cycle length at these three system configurations. This value gives the content freshness that a given system configuration can support for given request and database update rates. The figure shows the huge benefit provided by the proposed freshness-driven adaptive caching technique. The forth configuration consistently provides much fresher content than the two other configurations. Especially, during the heavy traffic conditions (i.e. during the periods of 10-20, 30-40, and 50-60 minutes), the system configuration with the freshness-driven adaptive caching supports content freshness up to 20 times better than those without dynamic content caching. It also supports content freshness up to 10 times better than even those systems that already deploy dynamic content caching solutions.

\subsection{Discussion}

Note that in some conditions, the second configuration (i.e. QT=60) performs better than the third configuration (i.e. QT $=140$ ); and vice verse in other conditions. On the other hand, the proposed freshness-driven adaptive caching technique will automatically adjust the number of cached query types to achieve the optimal assured content freshness. In Figure 8 we show the assured content freshness for the experimental results in 10-20, 30-40, and 50-60 minutes. The $\mathrm{X}$-axis is the number of cached query types and the Y-axis is the assured content freshness (i.e. the maximum of response time and invalidation cycle). We plot the assured content freshness at different user request rates and database update rates with respect to different numbers of 


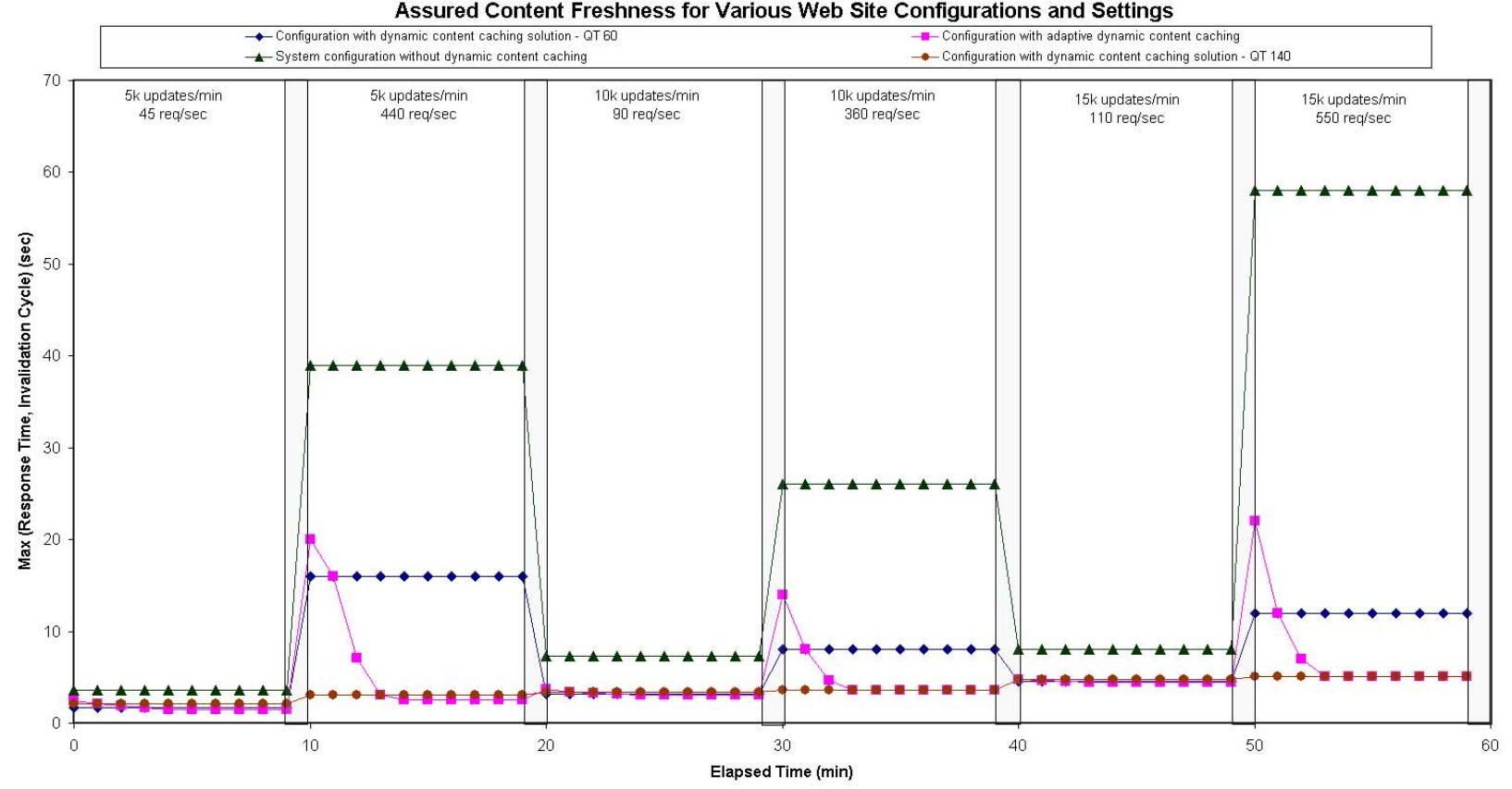

Figure 7. Comparisons of Assured Content Freshness for Four System Configurations

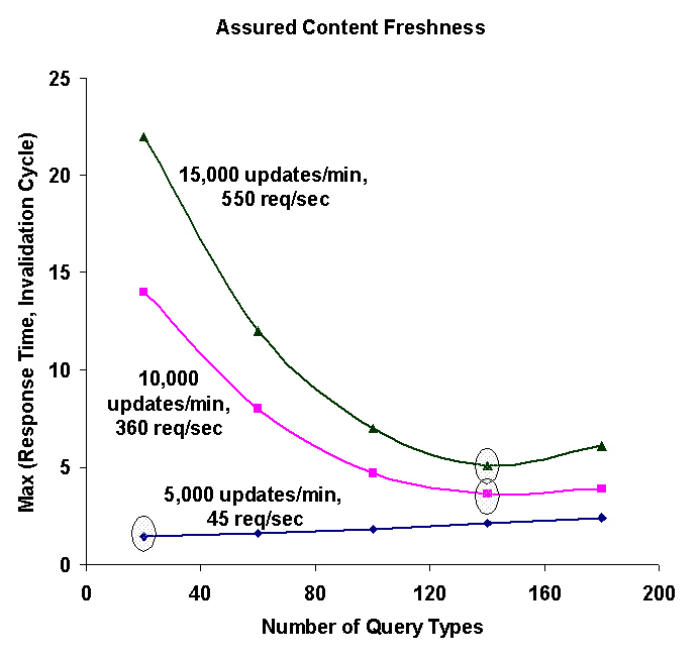

Figure 8. Effects of Adaptive Caching on Assured Content Freshness

cached query types at the cache servers. We can see that the curves of the assured content freshness are similar to the shape of a bowl. The best assured content freshness can be achieved at the bottom of the "cup" which is circled. The proposed freshness-driven adaptive caching technique continuously monitors response time and invalidation cycle to increase or to decrease the number of cached query types in the cache server so that the assured content freshness is maintained at the optimal point.

\section{Related Work}

Dynamai [12] from Persistence Software is one of the first dynamic caching solution that is available as a product. However, Dynamai relies on a proprietary software for both database and application server components. Thus it cannot be easily incorporated in existing e-commerce framework. IBM Research [13] has developed a scalable and highly available system for serving dynamic data over the Web. In fact, the IBM system was used at Olympics 2000 to post sport event results on the Web in timely manner. This system utilizes database triggers for generating update events as well as intimately relies on the semantics of the application to map database update events to appropriate Web pages. Other related works include [14], where authors propose a diffusion-based caching protocol that achieves loadbalancing, [15] which uses meta-information in the cachehierarchy to improve the hit ratio of the caches, [16] which evaluates the performance of traditional cache hierarchies and provides design principles for scalable cache systems, and [17] which highlights the fact that static client-to-server assignment may not perform well compared to dynamic server assignment or selection.

SPREAD[18], a system for automated content distribution is an architecture which uses a hybrid of client validation, server invalidation, and replication to maintain consistency across servers. This work focuses on static content and describes techniques to synchronize static content, rather than dynamic content. There has been various cache 
consistency protocol proposals which rely heavily on invalidation $[18,19,20]$. In our work, however, we concentrate on the updates of data in databases, which are by design not visible to the Web servers. Therefore, we introduce a vertical invalidation concept, where invalidation messages travel from database servers and Web servers to the front-end and edge cache servers as well as JDBC database access layer caches.

\section{Concluding Remarks}

Many e-commerce Web applications deploy dynamic content caching solutions to serve user requests at locations much close to users, avoiding network latency. In this paper, we propose a freshness-driven adaptive dynamic content caching technique. It monitors response time and invalidation cycle as feedback and dynamically adjusts caching policy. The technique aims at maintaining the best content freshness that a system configuration can support. By considering the trade-off of invalidation cycle and response time, our technique is able to maintain the best content freshness that can be assured, which occurs at the equilibrium point of response time and invalidation cycle. The experiments do show the effectiveness and benefits of the proposed freshness-driven adaptive caching. Under heavy traffic, the freshness-driven adaptive caching supports content freshness up to 20 times better than those without dynamic content caching. It also supports content freshness up to 10 times better than those systems that deploy dynamic content caching solutions without feedback.

\section{References}

[1] Zona Research. http://www.zonaresearch.com/.

[2] K. Seluk Candan, Wen-Syan Li, Qiong Luo, Wang-Pin Hsiung, and Divyakant Agrawal. Enabling Dynamic Content Caching for Database-Driven Web Sites. In Proceedings of the 2001 ACM SIGMOD Conference, Santa Barbara, CA, USA, May 2001. ACM.

[3] Wen-Syan Li, K. Seluk Candan, Wang-Pin Hsiung, Oliver Po, Divyakant Agrawal, Qiong Luo Wei-Kuang Wayne Huang, Yusuf Akca, and Cemal Yilmaz. Cache Portal: Technology for Accelerating Database-driven E-commerce Web Sites. In Proceedings of the 2001 VLDB Conference, Roma, Italy, September 2001.

[4] C. Mohan. Caching Technologies for Web Applications. In Proceedings of the 2001 VLDB Conference, Roma, Italy, September 2001.

[5] Mitch Cherniack, Michael J. Franklin, and Stanley B. Zdonik. Data Management for Pervasive Computing. In Proceedings of the 2001 VLDB Conference, Roma, Italy, September 2001.

[6] Anoop Ninan, Purushottam Kulkarni, Prashant Shenoy, Krithi Ramamritham, and Renu Tewari. Cooperative Leases:
Scalable Consistency Maintenance in Content Distribution Networks. Honolulu, Hawaii, USA, May 2002.

[7] Anindya Datta, Kaushik Dutta, Helen M. Thomas, Debra E. VanderMeer, Suresha, and Krithi Ramamritham. ProxyBased Acceleration of Dynamically Generated Content on the World Wide Web: An Approach and Implementation. In Proceedings of 2002 ACM SIGMOD Conference, Madison, Wisconsin, USA, June 2002.

[8] Qiong Luo, Sailesh Krishnamurthy, C. Mohan, Hamid Pirahesh, Honguk Woo, Bruce G. Lindsay, and Jeffrey F. Naughton. Middle-tier Database Caching for e-Business. In Proceedings of 2002 ACM SIGMOD Conference, Madison, Wisconsin, USA, June 2002.

[9] K. Selcuk Candan, Divyakant Agrawal, Wen-Syan Li, Oliver Po, and Wang-Pin Hsiung. View Invalidation for Dynamic Content Caching in Multitiered Architectures . In Proceedings of the 28th Very Large Data Bases Conference, Hongkong, China, August 2002.

[10] Wen-Syan Li, Wang-Pin Hsiung, Dmitri V. Kalashnikov, Radu Sion, Oliver Po, Divyakant Agrawal, and K. Selçuk Candan. Issues and Evaluations of Caching Solutions for Web Application Acceleration. In Proceedings of the 28th Very Large Data Bases Conference, Hongkong, China, August 2002.

[11] Wang-Pin Hsiung, Wen-Syan Li, K. Selçuk Candan, and Divyakant Agrawal. Multi-tiered Cache Management for ECommerce Web Sites. In Proceedings of Second International Workshop on Cooperative Internet Computing (CIC 2002), Hongkong, China, August 2002.

[12] Persistent Software Systems Inc. http://www.dynamai.com/.

[13] Eric Levy, Arun Iyengar, Junehwa Song, and Daniel Dias. Design and Performance of a Web Server Accelerator. In Proceedings of the IEEE INFOCOM'99, New York, New York, March 1999. IEEE.

[14] A. Heddaya, S. Mirdad, and D. Yates. Diffusion-based Caching: WebWave. In Proceedings of the 1997 NLANR Web Caching Workshop, 1997.

[15] M.R. Korupolu anf M. Dahlin. Coordinated Placement and Replacement for Large-Scale Distributed Caches. In Proceedings of the 1999 IEEE Workshop on Internet Applications, 1999.

[16] Renu Tewari and Michael Dahlin and Harrick M. Vin and Jonathan S. Kay. Design Considerations for Distributed Caching on the Internet. In Proceedings of the 19th International Conference on Distributed Computing Systems, 1999.

[17] R.L. Carter and M.E. Crovella. On the network impact of dynamic server selection. Computer Networks, 31(2324):2529-2558, 1999.

[18] P.Rodriguez and S.Sibal. Spread: Scaleable platform for reliable and efficient automated distribution. In Proceedings of the 9th World-Wide Web Conference, pages 33-49, Amsterdam, The Netherlands, June 2000.

[19] H. Yu, L. Breslau, and S. Shenker. A Scalable Web Cache Consistency Architecture. In Proceedings of the ACM SIGCOMM'99 Conference, Boston, MA, USA, September 1999.

[20] D. Li, P. Cao, and M. Dahlin. WCIP: Web Cache Invalidation Protocol, 2000. http://www.ietf.org/internetdrafts/draft-danli-wrec-wcip-00.txt. 